\title{
VARIACIÓN TEMPORAL DE LA ATENUACIÓN DE ONDAS SÍSMICAS EN LA REGIÓN DEL VOLCÁN PURACÉ, COLOMBIA
}

\section{TEMPORAL VARIATION IN THE SEISMIC WAVE ATTENUATION AT PURACÉ VOLCANO, COLOMBIA}

\author{
John Makario Londoñoํㅜ, Andrés Hernando Narváez ${ }^{2}$, Diana Marcela Quintero \\ *Email de correspondencia: jmakario@sgc.gov.co
}

Recibido: 11/08/2017

Aceptado: 15/01/2018

Publicado en línea: 20/04/2018

Citación: Londoño, J. M., Narváez, A. H. y Quintero, D. M. (2018). Variación temporal de la atenuación de ondas sísmicas en la región del volcán Puracé, Colombia. Boletín Geológico, 44, 75-88.

\section{RES UMEN}

Se realizó un estudio de atenuación de ondas sísmicas en la región del volcán Puracé (VP), Colombia, mediante el cálculo del inverso del parámetro coda $Q$ para sismos volcano-tectónicos registrados en el período 2006-2015. Para el cálculo de coda Q fueron seleccionados 310 sismos con localizaciones de alta calidad.

Los resultados mostraron que los valores de $Q$ en la zona del VP son, en general, bajos, lo que indica alta atenuación en dicha región. Adicionalmente, se relacionaron los datos de atenuación $\left(Q^{-1}\right)$ con valores de $V_{p} / V_{s}$ para determinar la relación $Q_{p}{ }^{-1} / Q_{s}{ }^{-1}$. Se encontraron valores bajos en la relación $Q_{p}{ }^{-1} / Q_{s}{ }^{-1}$, los cuales pueden estar asociados a la presencia de fluidos en fase líquida $y$, en menor proporción, a fluidos en estado gaseoso en la parte superficial del volcán, que afectan el sistema hidrotermal y que pueden ser fuentes generadoras de la sismicidad asociada a la actividad de fluidos (sismo tipo “tornillo", TO, y largo período, LP). Se sugiere que la actividad actual del VP está controlada por el sistema hidrotermal, con poca influencia del sistema magmático.

El parámetro $Q_{p}{ }^{-1} / Q_{s}{ }^{-1}$ puede ser usado como indicador de aumento o disminución del contenido de gas en el sistema hidrotermal del volcán, lo que lo convierte en una herramienta útil para el monitoreo rutinario del VP.

Palabras clave: Coda $Q$, atenuación ondas sísmicas, Vp/Vs, actividad sísmica, volcán Puracé, Qp $/ Q s, V p / V s$.

\footnotetext{
A B S T RACT

1 Ph. D. en Geofísica. Servicio Geológico Colombiano

2 Geógrafo. Servicio Geológico Colombiano

3 Geóloga. Servicio Geológico Colombiano
}

A seismic wave attenuation study was performed for volcano-tectonic earthquakes at Puracé volcano (PV), Colombia, for the period of 2006-2015 by using the inverse of coda $Q\left(Q^{-1}\right)$ of coda waves. A total of 310 high-quality earthquakes were used for the analysis. 
The results showed low $Q$ values for PV suggesting high attenuation in this region. Additionally, $Q$ values were related to $V_{p} / V_{s}$ data to calculate $Q_{p}{ }^{-1} / Q_{s}{ }^{-1}$ ratios. Low $Q_{p}{ }^{-1} / Q_{s}{ }^{-1}$ ratios were found, and they were associated with fluids predominantly in the liquid phase rather than in the gaseous phase located at shallow parts of the PV, which affected the hydrothermal system producing LP and "Tornillo"-type volcano earthquakes. It is inferred that the recent activity of PV is driven by the hydrothermal system, with a small influence from the magmatic system.

The $Q_{p}{ }^{-1} / Q_{s}{ }^{-1}$ ratio can be used as a marker for changes in gas content in the hydrothermal system, becoming a useful tool for routine monitoring tasks at PV.

Key words: Coda $Q$, seismic wave attenuation, $V p / V s$, seismicity, Puracé volcano, Qp/Qs.

\section{INTRODUCCIÓN}

a atenuación de las ondas sísmicas es un parámetro - sísmico importante para el estudio de la corteza terrestre y, en particular, para el estudio de zonas volcánicas. Este parámetro puede ser calculado mediante diferentes técnicas y métodos, siendo el más utilizado el que utiliza el parámetro conocido como coda Q. El método de Aki y Chouet (1975), conocido como "single scattering", o dispersor único, que asume la ubicación de la fuente y del receptor en el mismo sitio, se popularizó por su simplicidad y practicidad para el cálculo de la coda $Q$, cuyo inverso, $Q^{-1}$, es la atenuación de una señal sísmica en el tiempo. Posteriormente, Sato (1977) modificó este método y consideró la separación fuente-receptor, y lo denominó "isotropic single scattering", o dispersor único isotrópico.

Desde entonces, el cálculo de coda $Q$ ha sido aplicado en diferentes zonas del mundo para conocer la atenuación de las ondas sísmicas, particularmente en la corteza terrestre, donde se presentan diferentes ambientes tectónicos y geológicos (Roecker et al., 1982; Singh y Hermann, 1983; Pulli, 1984; Gusev y Lemzikob, 1985; Phillips y Aki, 1986; Ambeh y Fairhead, 1989; Kvamme y Havskov, 1989). Algunos estudios han sugerido que la coda Q varía antes de la ocurrencia de sismos y erupciones volcánicas, y que como tal podría considerarse como un premonitorio (Jin y Aki, 1986; Fehler et al., 1988; Londoño, 1996; Tusa et al., 2004; Giampicolo et al., 2007). En la industria petrolera ha sido una práctica común usar la atenuación sísmica como un poderoso parámetro para diferenciar gas de petróleo (Prasad et al., 2004; Zhand y Stewart, 2007; Hermana et al., 2014).

Diferentes estudios de atenuación sísmica se han llevado a cabo en zonas volcánicas para conocer tanto la es- tructura interna como para detectar variaciones temporales de este parámetro asociados a cambios en la actividad volcánica (Ortiz et al., 1992; Bianco et al., 1999; Del Pezzo et al., 1996; Wegler y Luhr, 2001; Londoño y Sudo, 2002; Del Pezzo, 2008; De Siena et al., 2014). Se ha establecido que el valor de coda $Q$, para muchas regiones, es muy similar o cercano al componente de absorción intrínseca del medio ( $Q$ intrínseco) para ciertas bandas de frecuencia (Londoño, 1996; Londoño y Sudo, 2002), razón por la cual es muy sensible a cambios de temperatura, presión de poros o presencia de gas o fluidos en el medio, aspecto que hace que el cálculo de coda $Q$ sea muy apropiado para zonas volcánicas (Gao, 1992; Del Pezzo, 2008). Las variaciones temporales de este parámetro pueden ayudar en la interpretación y comprensión de la actividad volcánica, especialmente en modificaciones del medio debidas tanto a actividad hidrotermal o magmática (Fehler et al., 1988; Londoño y Sudo, 2002; Yamamoto y Sato, 2010; De Siena et al., 2014), como a las variaciones en el campo de esfuerzos (Jin y Aki, 1986; Guo et al., 2009).

En Colombia se han realizado varios estudios sobre coda $Q$ en zonas volcánicas, particularmente en el volcán Nevado del Ruiz y en el volcán Galeras, dos de los más activos de dicho país (Londoño, 1996; Londoño y Sudo, 2002; Lacruz et al., 2009; Vargas et al., 2012). El volcán Puracé (VP) es un estrato volcán activo (4650 msnm) que se encuentra localizado en el departamento del Cauca, Colombia, en las coordenadas geográficas $2^{\circ} 19^{\prime} 01 \mathrm{~N} \mathrm{y}$ $76^{\circ} 23^{\prime} 53 \mathrm{~W}$, a una distancia de $26 \mathrm{~km}$ al SE de Popayán. Es el extremo noroccidental de la cadena volcánica de los Coconucos, compuesta por quince centros eruptivos alineados con una orientación $\mathrm{N} 39^{\circ} \mathrm{W}$, la mayoría de ellos sobre el trazo de la falla Coconucos. Su edificio tiene forma de cono truncado con laderas de $30^{\circ}$ de in- 
clinación, presenta un cráter interno y otro externo, concéntricos, de 500 y $900 \mathrm{~m}$ de diámetro, respectivamente. La actividad fumarólica se concentra en el interior del cráter, principalmente en una gran grieta que atraviesa el fondo; también hay un gran campo fumarólico en el flanco externo NW del volcán (fumarola lateral). Estudios geológicos recientes revelan una historia eruptiva caracterizada por actividad predominantemente explosiva, cuyos productos principales han sido lavas andesíticas, flujos piroclásticos, piroclastos de caída y flujos de lodo. Esta actividad ha sido reportada a partir de 1801, y se han descrito desde 1827 al menos quince episodios eruptivos bien documentados, que han ocasionado daños materiales y pérdidas de vidas. La última erupción menor considerada ocurrió en marzo de 1977, asociada a salida de cenizas (Espinosa, 2001). En la zona se observan dos sistemas de fallas: sistema de fallas de Moras y sistema de fallas Coconucos. El primero tiene una dirección NE y cruza la base del edificio volcánico del VP; el segundo, con dirección NW, similar a la orientación de la cadena volcánica (Monsalve, 1996; Monsalve y Pulgarín, 1999).

En la actualidad el VP presenta actividad sísmica permanente, además de la presencia de una columna de vapor y gases. En la última década se ha incrementado su actividad sísmica, caracterizada por la ocurrencia de sismos volcano-tectónicos y sismos asociados a actividad de fluidos, particularmente de tipo "tornillo" (figura 1). Este estudio se enfoca en el análisis temporal de la atenuación sísmica en el VP y su relación con la actividad, con miras a aumentar el conocimiento de dicho volcán y a contribuir a mejorar las tareas de monitoreo continuo.

\section{MÉtOdo, DATOS Y PROCESAMIENTO}

El método usado en este estudio para calcular la atenuación de las ondas sísmicas (inverso de $Q, o Q^{-1}$ ) asume que las ondas coda son ondas $S$ y ondas $S$ retrodispersadas desde heterogeneidades distribuidas aleatoriamente (Aki y Chouet, 1975). Además, se considera la separación entre la fuente sísmica y la estación o receptor, que es básicamente la diferencia entre los métodos de Aki y Chouet (1975) y Sato (1977). Una forma linealizada del modelo de dispersión simple de Sato (1977) se puede escribir así:
$b=\frac{w}{Q} \quad k\left(\frac{t}{t_{s}}\right)=\left(\frac{t}{t_{s}}\right) \ln \left|\frac{t+t_{s}}{t-t_{s}}\right|$

donde

As es la máxima amplitud de las ondas $S, A(t)$ es la amplitud promedio tomada en el tiempo $t$ y medida desde el origen del sismo, $t_{s}$ es el tiempo de viaje de la onda $S, w$ es la frecuencia angular, $C$ es una constante y $Q$ es el factor de calidad. A partir de la pendiente $b$ es posible calcular $Q$ por medio del ajuste de la ecuación (1), usando técnicas de mínimos cuadrados. La figura 1 muestra un ejemplo del cálculo de coda $Q$.

El monitoreo de la actividad del volcán Puracé se viene realizando de forma permanente, en tiempo real, desde hace veinte años. El volcán cuenta actualmente con una red sismológica telemétrica compuesta por siete estaciones sísmicas. Las estaciones COND y MINA se seleccionaron para el cálculo de coda $Q$ por poseer el registro más continuo en el tiempo, tener mejor calidad de la señal y estar ubicadas relativamente cerca y en el mismo sector del volcán y con condiciones de sitio similares (Raigosa, 2017, comunicación personal) para evitar posibles cambios en coda $Q$ asociados a la ubicación de la estación (figura 2). Los sismos usados para la realización de este trabajo corresponden a la base de datos del volcán Puracé del Servicio Geológico Colombiano (SGC) en el período 2006-2015 (figura 3). Se seleccionaron 310 sismos volcano-tectónicos (VT) de alta calidad para el cálculo de coda $Q$ (figura 4) que cumplieron con los siguientes criterios de selección: localización hipocentral con RMS menor a $0,1 \mathrm{~s}$ y error horizontal y vertical menor a $1,0 \mathrm{~km}$; duración mayor a $20 \mathrm{~s}$, sismos en un rango de profundidades entre 0 y $12 \mathrm{~km}$ cuya relación señal/ruido de la coda fuera superior a 2, y que no estuvieran saturados.

Siguiendo la metodología de Londoño (1994), se calculó el valor de coda $Q$ en las bandas de frecuencias centradas en 6 y $12 \mathrm{~Hz}$ aplicando un filtro pasa banda Butterworth, y se tomó el RMS de la porción de la coda a partir de dos veces el tiempo de viaje de la onda $S$, en ventanas de 1,5 s hasta donde el RMS de la amplitud de la coda del sismo en esa ventana fuera superior a dos veces el ruido de fondo (figura 1). Para el análisis de los resultados se usó la atenuación, o el inverso de Coda $Q, Q^{-1}$. La escogencia de los rangos de frecuencias se basa en trabajos anteriores en zonas volcánicas y en el enfoque que 
tiene este trabajo, que busca encontrar cambios en la atenuación debidos a heterogeneidades del orden de decenas de metros a un par de centenares de metros, que corresponderían a las frecuencias seleccionadas (centradas en 6 y $12 \mathrm{~Hz}$ ). Otros trabajos sobre coda $Q$ en zonas volcánicas se han realizado usando bandas de frecuencia similares a las analizadas en este estudio (Fehler et al., 1988; Londoño y Sudo, 2002; Yamamoto y Sato, 2010; De Siena et al., 2014). Por otra parte, se escogieron promedios mensuales para el análisis estadístico de los datos, siguiendo la metodología usada por Londoño y Sudo (2002) para el volcán Nevado del Ruiz; ellos establecieron que en una ventana (promedio) de treinta días se podían observar más claramente variaciones temporales de coda $Q$ asociadas a cambios en las condiciones internas del volcán y en el contenido de gas, o a la relación de aspectos de los poros, etc., en lugar de promedios quincenales, semanales o de otro lapso de tiempo. No obstante, vale la pena mencionar que se podrían usar otros rangos de tiempo, pero en este trabajo se escogieron promedios mensuales.
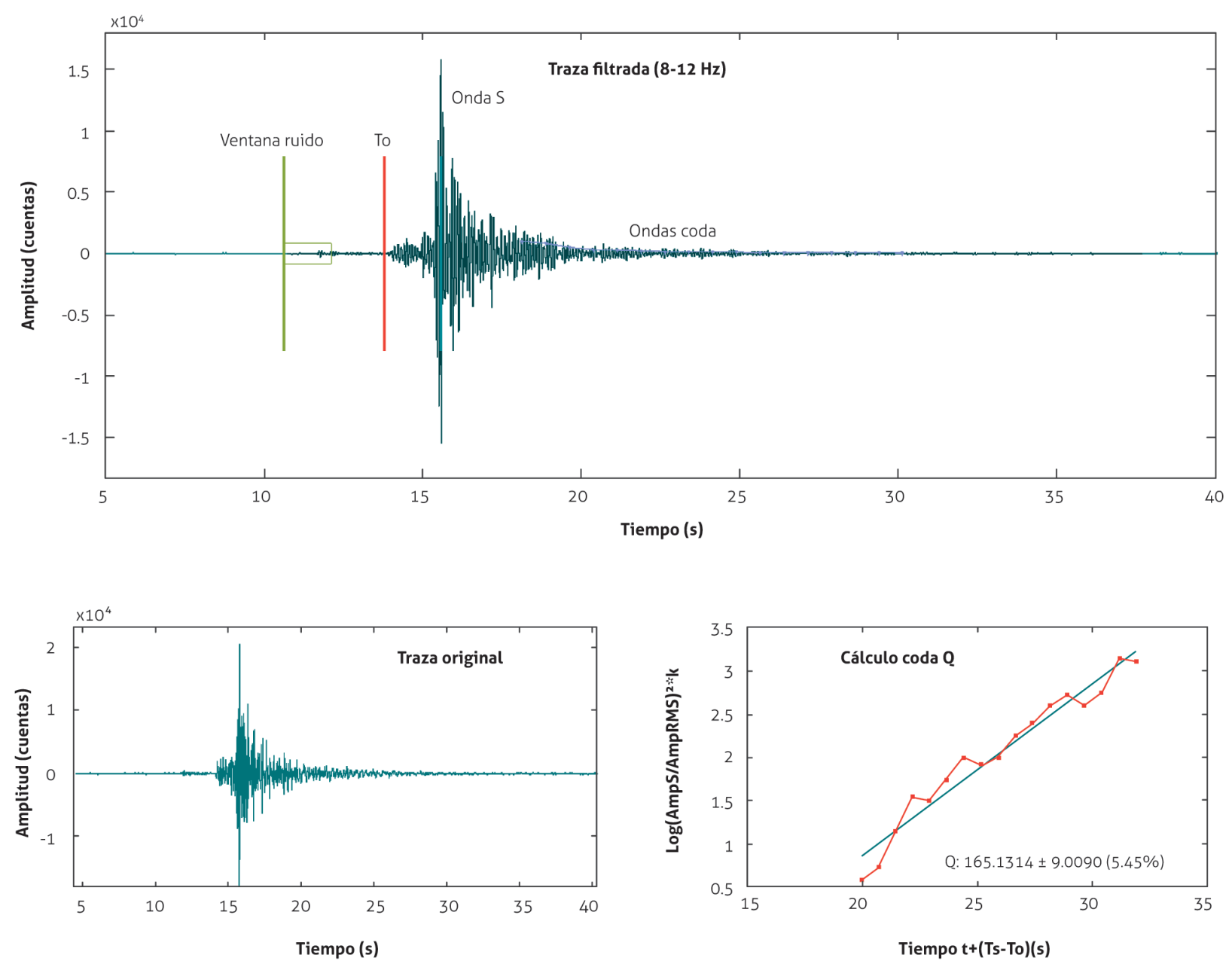

Figura 1. Ejemplo de cálculo de coda Q para un sismo VT en el volcán Puracé. En la ventana inferior izquierda se muestra la traza original. En la ventana superior se muestra la traza filtrada usando un filtro pasa banda Butterworth, en la banda de frecuencias centrada en $6 \mathrm{~Hz}$. $T_{0}=$ tiempo de origen del sismo. La línea fucsia en la ventana superior representa la porción denominada ondas coda (porción de la señal sísmica después de dos veces el tiempo de viaje de la onda $S$, iniciando en $T$ ). La ventana de ruido de fondo (barra y recuadro verde) se usó para delimitar hasta dónde se registran las ondas coda (mayor a 2 veces el RMS de la amplitud de la ventana de ruido). El cálculo de $Q$ se realiza ajustando la curva del logaritmo de los valores de RMS de la amplitud corregidos por la distancia fuente-receptor versus el tiempo transcurrido ("lapse time") desde dos veces el tiempo de viaje de la onda $\mathrm{S}$, y calculando la pendiente de dicho ajuste (ventana inferior derecha)

Fuente: autores 


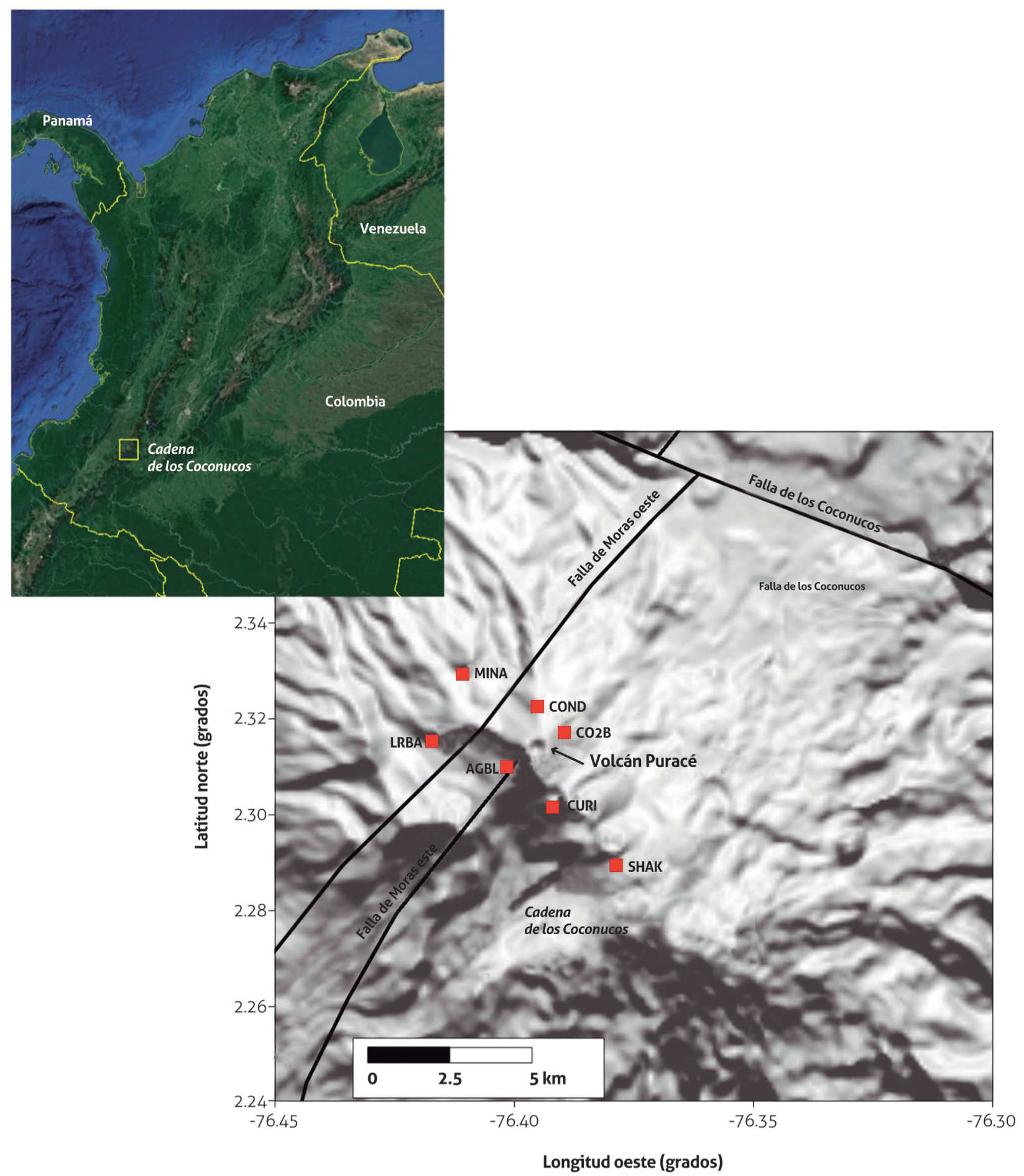

Figura 2. Mapa de localización y red sísmica actual (cuadros rojos) del volcán Puracé. Las estaciones COND y MINA se usaron para el análisis de coda $Q$. Las líneas negras gruesas representan fallas geológicas

Fuente: autores. Imagen satelital: Google.com 


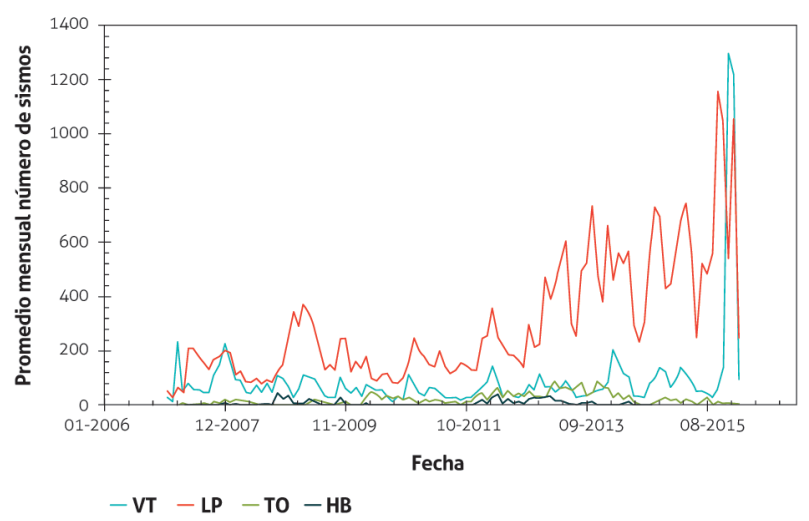

Figura 3. Promedios mensuales de series de tiempo de número de sismos volcánicos ocurridos en el volcán Puracé, 2006-2015. $\mathrm{VT}=$ volcano-tectónico, $\mathrm{LP}=$ largo período, $\mathrm{TO}=$ sismos tipo "tornillo", HB = sismos híbridos (mezcla de VT y LP) Fuente: autores
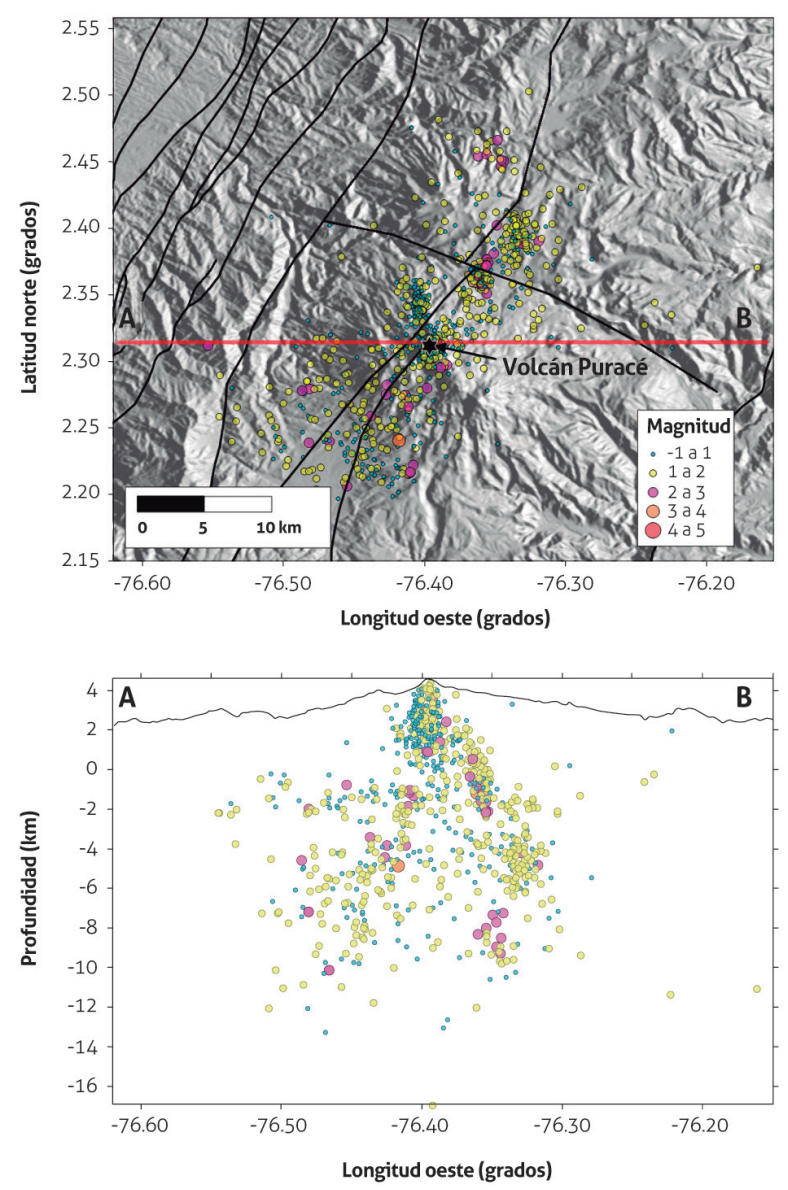

Figura 4. Localización de sismos VT del volcán Puracé usados para el análisis de coda Q. Arriba, epicentros; abajo, hipocentros mostrados según perfil AB. Los triángulos representan estaciones sísmicas, y la estrella, la localización del cráter del VP. Las líneas gruesas negras representan fallas geológicas

gruesas negra
Fuente: autores

\section{Resultados}

Antes de realizar cualquier análisis de los resultados obtenidos se verificó que las posibles variaciones temporales de $Q^{-1}$ no se debieran a efectos de la localización hipocentral (Londoño y Sudo, 2002). Se hicieron pruebas en las que se graficó la latitud, la longitud y la profundidad con el tiempo, y no se observó ninguna tendencia, lo que confirma que si se registran cambios temporales en $Q^{-1}$ en el VP, se deben a una causa diferente a la ubicación espacial (fig. 5). Por otra parte, se analizó la influencia de la banda de frecuencias usada sobre los resultados de $Q^{-1}$. La figura 6 muestra la comparación entre las series de tiempo de $Q^{-1}$ en las frecuencias centradas en 6 y $12 \mathrm{~Hz}$ en las estaciones COND y MINA. No se observaron mayores variaciones entre una banda y otra en ambas estaciones. La correlación entre bandas de frecuencia arrojó un coeficiente de correlación $r=0,65$ en la estación COND, y $r=0,55$ en la estación MINA. De acuerdo con trabajos anteriores realizados en zonas volcánicas, la banda de frecuencia centrada en $12 \mathrm{~Hz}$ es apropiada para analizar posibles cambios en coda $Q$ asociados a cambios internos en el volcán debidos a absorción intrínseca del medio y a dispersores de tamaño pequeño (Fehler et al., 1988; Londoño, 1994; Londoño y Sudo, 2002); por tal razón, se escogió la banda de frecuencia centrada en $12 \mathrm{~Hz}$ para el análisis de coda $Q$ en el VP.

La figura 7 muestra la variación temporal de $Q^{-1}$ de cada sismo seleccionado en el período 2006-2015, ocurridos en el volcán Puracé y registrados en las estaciones COND y MINA en la banda de frecuencias centrada en $12 \mathrm{~Hz}$. En esta figura se puede observar que los valores de $Q^{-1}$ varían en un amplio rango, entre 0,0025 y 0,017, y que no hay una tendencia temporal. Con miras a detectar un posible cambio o tendencia más general, se procedió a calcular el promedio mensual de $Q^{-1}$. La figura 8 muestra la comparación del promedio mensual de $Q^{-1}$ con el número mensual de sismos asociados a la actividad de fluidos en el sistema volcánico (tipo largo período, LP + TO). 

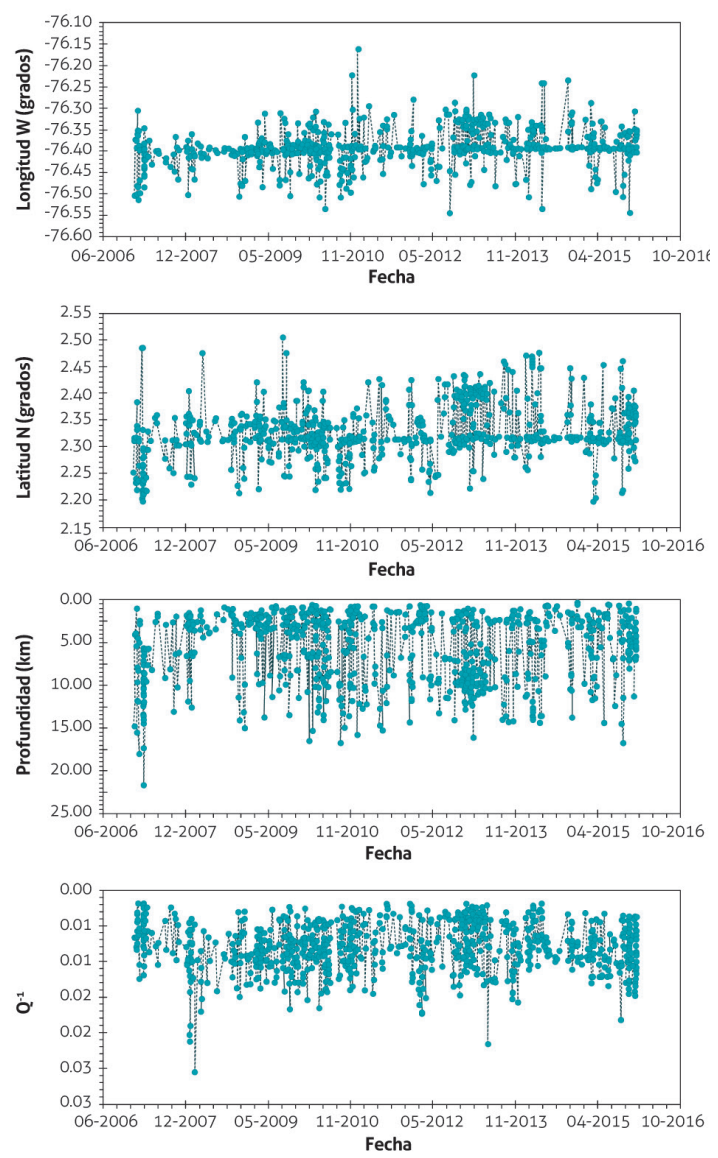

Figura 5. Comparación temporal de la localización hipocentral (latitud, longitud, profundidad) y $Q^{-1}$ en la estación COND Fuente: autores
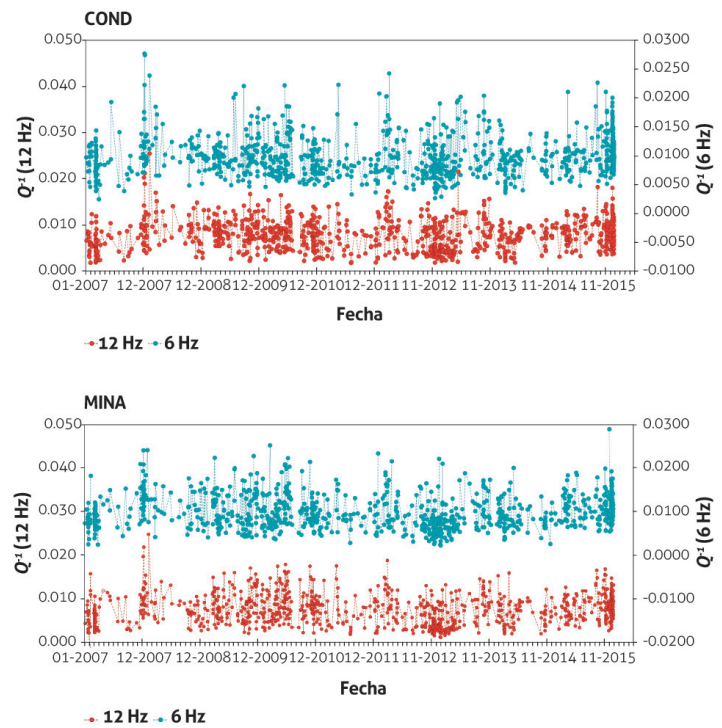

Figura 6. Comparación de series de tiempo de valores de coda $Q$ en las bandas de frecuencia centradas en 6 y $12 \mathrm{~Hz}$ en las estaciones COND y MINA

Fuente: autores

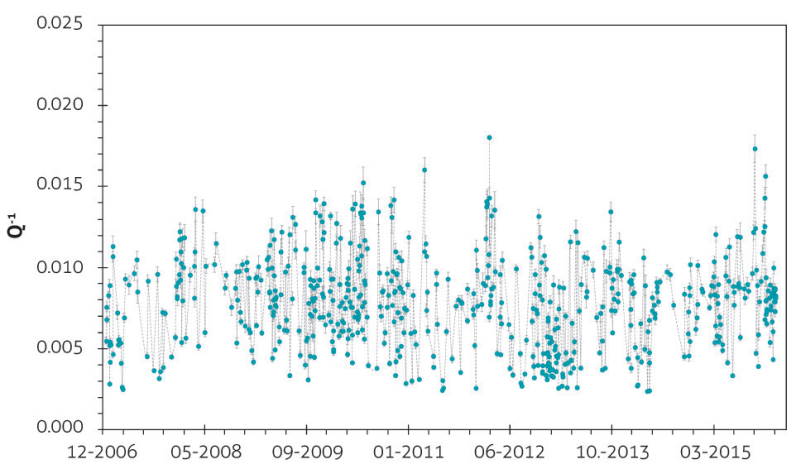

Figura 7. Variación temporal de $Q^{-1}$ para el volcán Puracé. Promedio de $Q^{-1}$ de cada sismo analizado en las estaciones COND y MINA con la banda de frecuencias centrada en $12 \mathrm{~Hz}$. Las líneas verticales sobre los picos representan el error estándar. Nótese que los valores de los errores son bajos, menores al $10 \%$

Fuente: autores

En esta figura es posible observar un comportamiento oscilatorio de $Q^{-1}$ a lo largo del tiempo sin presentar una relación clara con el número de sismos LP, aun cuando la ocurrencia de estos se incrementó en promedio en un poco más del doble a partir de agosto de 2012. Por otra parte, parecen existir algunas variaciones temporales significativas de $Q^{-1}$. Estos cambios en $Q^{-1}$ se representan en la figura 8 con franjas de colores, cuyos valores están entre dos niveles, uno por encima del promedio más la desviación estándar de $Q^{-1}$, y otro por debajo del promedio más o menos la desviación estándar de $Q^{-1}$.

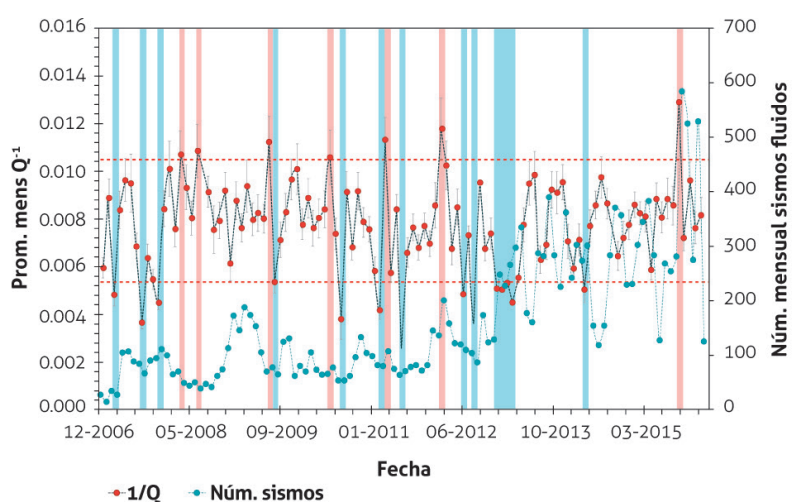

Figura 8. Promedio mensual de $Q^{-1}$ del volcán Puracé, y relación con el número mensual de sismos tipo asociados con fluidos $(\mathrm{LP}+\mathrm{TO})$. Las líneas rojas punteadas horizontales representan los valores del promedio de $Q^{-1}$ más la desviación estándar (superior) y el promedio de $Q^{-1}$ menos la desviación estándar (inferior); las barras coloreadas representan los valores de $Q^{-1}$ por debajo (azul) y por encima (café) de dichos valores. Las líneas verticales sobre los picos representan el error estándar

Fuente: autores 


\section{Análisıs}

De acuerdo con los resultados obtenidos, se puede concluir que no hay una correlación clara de los valores de la coda $Q$ y la actividad sísmica asociada a fluidos en el VP en el período estudiado. Esto puede explicarse por los niveles relativamente bajos de actividad sísmica de fluidos en este volcán, que no desestabilizaron el sistema hidrotermal o magmático, o porque el medio no presentó variaciones significativas, ya que permaneció muy estable en el sentido de no mostrar tendencias de cambio paulatino a lo largo del tiempo. Aunque el comportamiento del medio fue estable en general, algunas veces pudo presentar cambios temporales cortos que dieron lugar a las variaciones previamente mencionadas, sin afectar de forma significativa la actividad del volcán.

Con miras a observar relaciones entre $Q^{-1}$ y otros parámetros sísmicos, se procedió a calcular la relación $V_{p} / V_{s}$ de los mismos sismos usados para el cálculo de $Q^{-1}$, usando el método de Wadatti modificado, que consiste en graficar la relación del tiempo de viaje de la onda $S$ versus el tiempo de viaje de la onda $P$, asumiendo una relación de Poisson constante, de manera que se formará una línea recta con pendiente igual a $\left(V_{p} / V_{s}\right)-1$. En este método se deben seleccionar estaciones sísmicas y sismos localizados en ciertas regiones específicas para obtener valores más confiables de $V_{p} / V_{s}$ y evitar el efecto de la distribución espacial de los sismos y estaciones cuando se calcula este valor, sin discriminar por fuentes o grupos de estaciones (véanse detalles del método en Chatterjee et al., 1985). El método fue aplicado para la fuente sismogénica cercana al volcán Puracé, y se seleccionaron las estaciones y los sismos que se muestran en la figura $9 \mathrm{~b}$ para el cálculo del $V_{p} / V_{s}$. La figura 9a muestra la variación temporal del promedio mensual de $Q$ y $V_{p} / V_{s}$ en dicha fuente. No se observa una tendencia o correlación clara entre ambos parámetros, aunque en algunos períodos se observa la típica relación inversa (Mavko et al., 2005). Para observar una mejor relación entre estos dos parámetros se graficó la relación $Q / V_{p} / V_{s}$. La figura 10 muestra la variación temporal individual y el promedio mensual de la relación entre $Q / V_{p} / V_{s}$ de los sismos seleccionados comparados con la energía sísmica radiada en cada sismo
LP disponible (calculada usando la fórmula de Dibble, 1974 y el método de Alparone et al., 2003). En la figura $10 \mathrm{~b}$ se puede observar que no parece existir una correlación con la energía sísmica, lo que sugiere que la relación $\mathrm{Q} / V_{p} / V_{s}$ es independiente de ella $(r=0,05)$. No obstante, la relación $Q / V_{p} / V_{s}$ mostró un aparente comportamiento oscilatorio con algunas variaciones temporales: se observa una disminución paulatina (más de tres meses seguidos) en los períodos diciembre de 2008 a marzo de 2009, noviembre de 2010 a febrero de 2011, octubre de 2012 a febrero de 2013, y diciembre de 2014 a junio de 2015 (fig. 10b). Estas variaciones temporales de $Q / V_{p} / V_{s}$ pueden estar asociadas a cambios en las condiciones internas (cambio de fase) de los fluidos en la parte superficial del volcán (Zhang y Stewart, 2007; Hermana et al., 2014; Qi et al., 2017), sin que tenga un efecto en la energía, o desplazamiento reducido, DR, o la cantidad de sismos tipo LP.

Para comprobar esta hipótesis se procedió a calcular la variación temporal de la relación del inverso de $Q$ para la onda $P, Q_{P}$, sobre el inverso de $Q$ para la onda $S, Q_{S}$, $Q_{P} / Q_{S}$ a partir de los valores obtenidos de $V_{p} / V_{s}$ mediante la formulación de Zhang y Stewart (2010) y Mavko et al. (2005). Asumiendo que la coda $Q(Q)$ es igual al valor de $Q$ para la onda $S\left(Q=Q_{S}\right)$ se tiene:

$$
\frac{Q p^{-1}}{Q s^{-1}}=\frac{\frac{5(M(G-2))^{2}}{4(M /(G-1))}}{2\left(\frac{M}{G}+\frac{M}{G}\right)} \frac{1(3 M /(G-2)) 3(M / G-1))}{(G-2)}
$$

Donde $M$ y $G$ son los módulos de compresión y cizalla que se pueden obtener a partir de los valores de $V p / V s$ mediante la siguiente relación:

$$
\frac{M}{G}=\frac{2-2 v}{1-2 v}=\frac{V_{p}^{2}}{V_{s}^{2}}
$$

Donde $v$ es la relación de Poisson. Si se usa la formulación de Mavko et al. (2005), la ecuación (2) se puede reescribir en términos de $V_{p} / V_{s}$ :

$\frac{Q p^{-1}}{Q s^{-1}}=\frac{1}{4} \frac{\left(\left(V_{p} / V_{s}\right)^{2}-2\right)^{2}\left(3 \times\left(V_{p} / V_{s}\right)^{2}-2\right)}{\left(\left(V_{p} / V_{s}\right)^{2}-1\right) \times\left(V_{p} / V_{s}\right)^{2}}$ 
a)

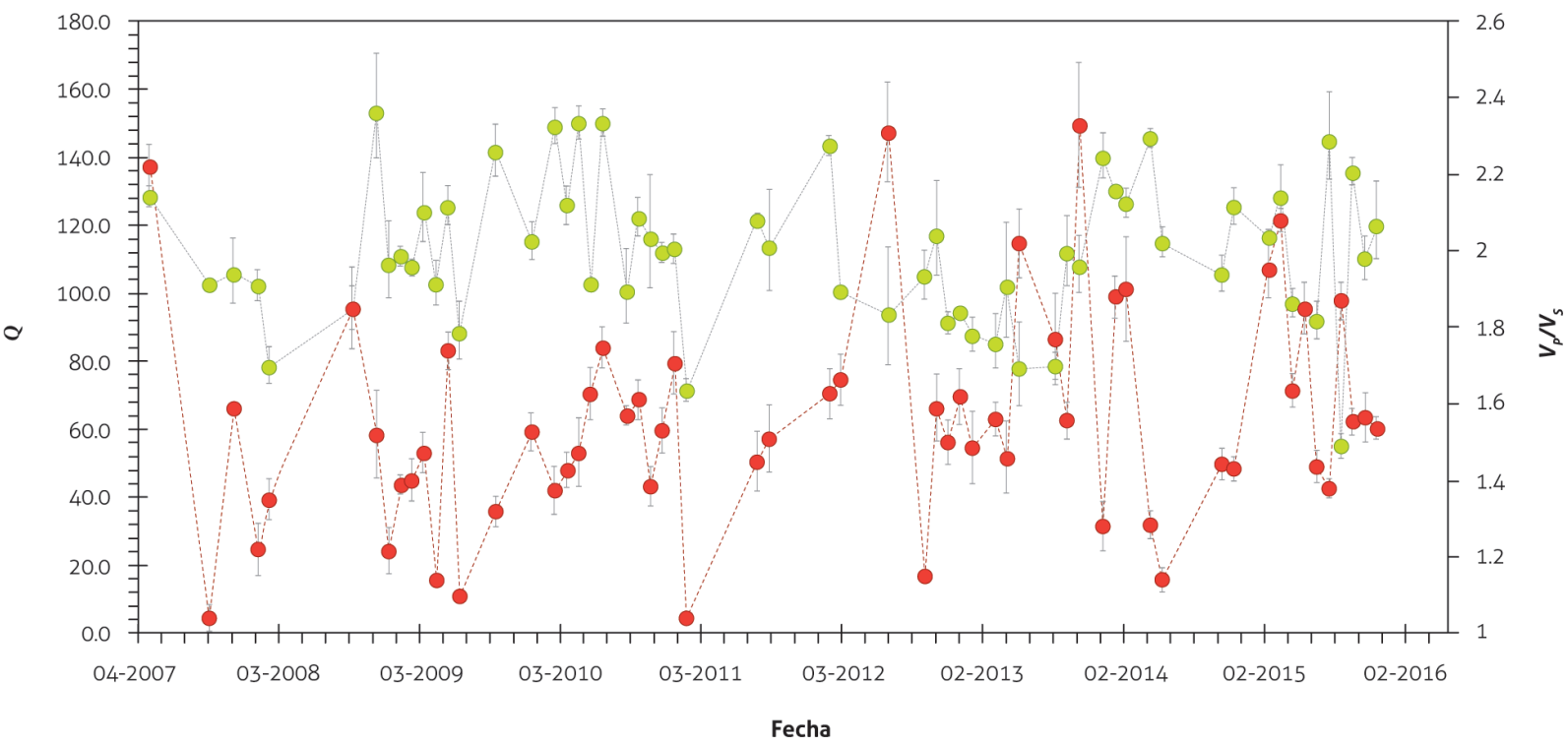

O $\quad-\cdots$ Vp/Vs

b)
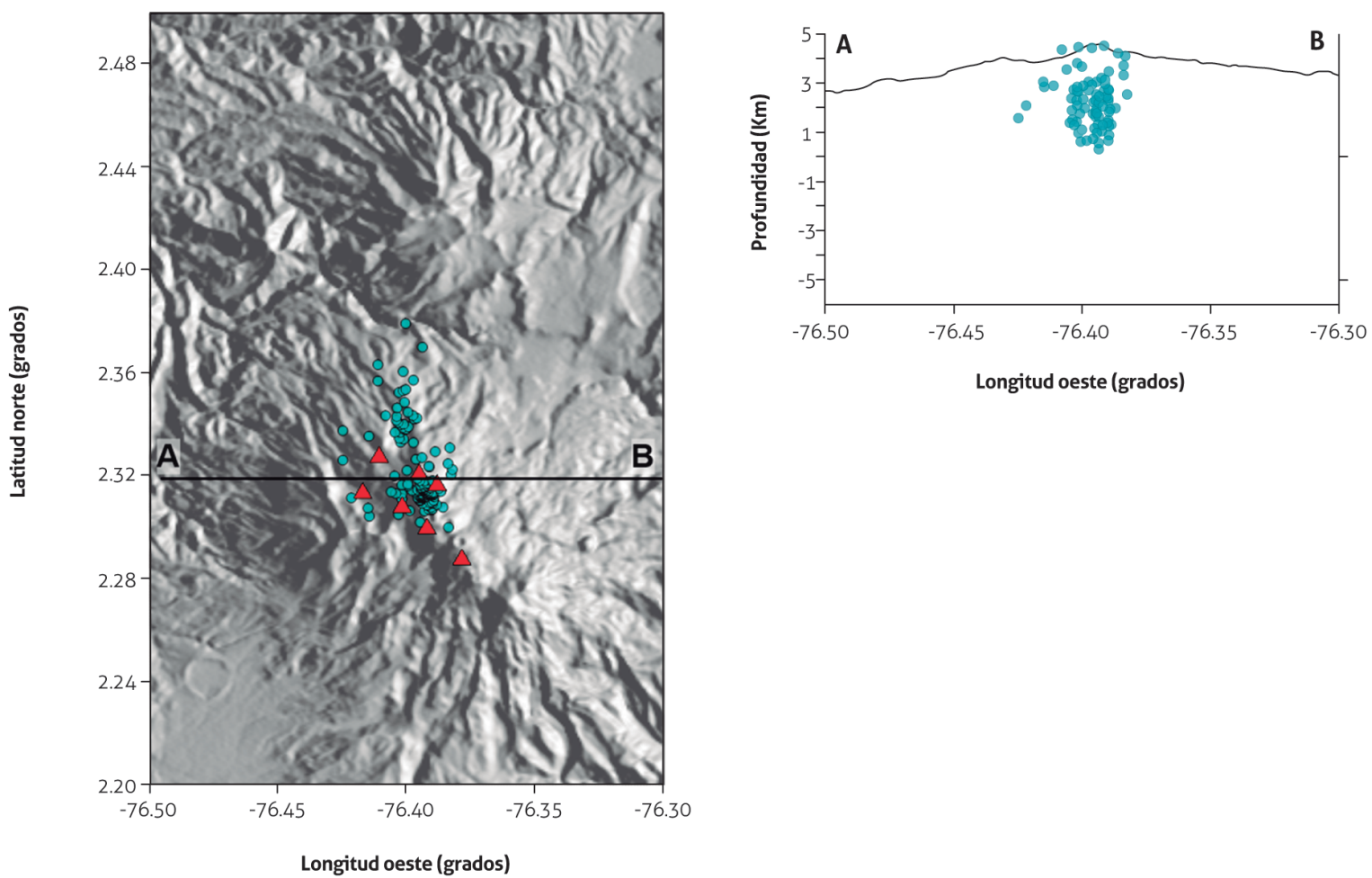

Figura 9. (a) Relación entre el promedio mensual de $Q$ y el promedio mensual de la relación $V / V$ en la fuente sismogénica cercana al cráter del volcán Puracé. (b) Localización de dicha fuente tanto en planta como en perfil. Las líneas verticales sobre los picos representan el error estándar de $Q$ y $V_{p} / V_{s}$. La línea $\mathrm{AB}$ representa el perfil EW

Fuente: autores 
La figura 11 muestra los valores de $Q_{p}^{-1} / Q_{s}^{-1}$ versus el desplazamiento reducido calculado para ondas superficiales de los eventos tipo tornillo (TO). Se puede observar un comportamiento inverso en algunas épocas entre esta relación y la ocurrencia de sismos (aumento de la energía) asociados a actividad de fluidos (sismos tipo "tornillo", $\mathrm{TO})$, y en otras épocas no parece haber correlación alguna (coef. de correl., $r=0,15)$. Valores altos de $Q_{p}{ }^{-1} / Q_{s}^{-1}$ se han relacionado con aumento en el contenido de fluidos en fase gaseosa en el interior de la corteza (Mavko et al., 2005) o con aumento del tamaño de los poros o aumento de gas en ambientes petrolíferos (Zhang y Stewart, 2010). Qi et al. (2017) recientemente propusieron que valores de $Q_{p}^{-1} / Q_{s}^{-1}$ mayores a uno están asociados con presencia de gas, mientras que valores menores a uno se asocian con presencia de agua o fluidos en fase líquida. Para el caso del VP, en su gran mayoría los valores de $Q_{p}^{-1} / Q_{s}^{-1}$ en el período estudiado fueron menores a uno (88\%). Esto podría indicar que en el interior del VP, en la zona que mapean los sismos VT escogidos, predominan fluidos en una fase líquida sobre una fase gaseosa, que puede ser la principal generadora de sismos asociados a esa actividad de fluidos (tipo "tornillo", TO, y tipo largo período, LP). Una evidencia de esta suposición es la escasa cantidad de gas que sale hacia la atmósfera a través de las fumarolas que actualmente tiene el cráter activo, aunque haya habido un incremento de la sismicidad tipo LP y TO durante algunas épocas del período estudiado (SGC, 2016); es posible que en la parte superficial del edificio volcánico se acumule temporalmente poca cantidad de gas que luego es liberado a la atmósfera a través de la fumarola, mientras que la cantidad de agua es proporcionalmente mucho mayor al gas, y se manifiesta con un gran número de fuentes termales, tanto cerca como lejos del cráter activo, a la vez que en la actividad sísmica tipo LP y TO.

Esto permite sugerir que la relación $Q_{p}^{-1} / Q_{s}^{-1}$ podría servir como parámetro indicador de la fase en la que se encuentran los fluidos del sistema hidrotermal (líquida o gaseosa) en la parte superficial del edificio volcánico.

Aunque este volcán no ha hecho erupción en el tiempo durante el cual se lo ha monitoreado con instrumentos, ha presentado algunos cambios en su actividad sísmica, particularmente con la ocurrencia de sismos tipo "tornillo" (TO). Estos sismos presentaron varios incrementos en el período estudiado, y en particular un incremento sosteni- do entre septiembre de 2012 y noviembre de 2013 (figuras 10 y 11). Este período mostró valores $Q_{p}^{-1} / Q_{s}^{-1}$ que variaron en un amplio rango sin mostrar una tendencia clara. Es posible que esta actividad pueda asociarse a la combinación de fluidos hidrotermales en fase gaseosa y líquida en la parte superficial del volcán, probablemente sin ningún aporte de material magmático, a juzgar por los resultados de muestreos geoquímicos en la fumarola y fuentes termales que no muestran componentes magmáticos ni cambios en la deformación volcánica que indiquen aporte de magma en este período en el VP (SGC, 2016). Un estudio reciente de $Q$ radiado $(Q r)$, por medio del método Sompi con base en frecuencias complejas y el modelo de la grieta (Kumagai y Chouet, 2002) para sismos TO del VP parece soportar esta conclusión: el estudio encontró valores altos de $Q r$ (desde 100-400 en promedio hasta 800), cuyo origen, según las interpretaciones, se debe a la presencia de material particulado (azufre nativo proveniente de los mantos de azufre explotados en una mina cercana) mezclado con poco gas en estos sismos TO (Alpala et al., 2014). Asimismo, el rango de valores $Q r$ que predominaron en esos sismos (100-300) corresponde, según Kumagai et al. (2002), a agua con gas. Por otra parte, los valores de $Q_{p}^{-1} / Q_{s}^{-1}$ obtenidos corresponden a sismos VT localizados entre 0 y $4 \mathrm{~km}$ de profundidad (figura $9 \mathrm{~b}$ ). Por lo tanto, se puede suponer que las principales variaciones de $Q_{p}^{-1} / Q_{s}^{-1}$ se deben a cambios en el medio en la parte superficial del volcán (sistema hidrotermal) más que a cambios en el medio ocurridos a mayores profundidades que pudieran estar asociadas con actividad magmática. Esta suposición se soporta en el hecho de que no hay evidencias de nuevo aporte magmático en el tiempo que lleva el monitoreo instrumental en dicho volcán. Es evidente que el VP emite poca cantidad de gases magmáticos a la atmósfera, de acuerdo con las esporádicas mediciones de $\mathrm{SO}_{2}$ disponibles que se han realizado con DOAS móvil, y a las continuas con Scan DOAS, que no superan las 50 t/día en promedio (SGC, 2017); igualmente, los valores de emisión de $\mathrm{CO}_{2}$ en la fumarola principal son bajos en general. La figura 12 muestra las series de tiempo de los valores de $Q_{p}^{-1} / Q_{s}^{-1}$ y la concentración de $\mathrm{CO}_{2}$ en el período comprendido entre 2013 y 2015, que corresponde al lapso del que se dispone de mediciones continuas de $\mathrm{CO}_{2}$ en la fumarola principal del VP. Como se puede observar en dicha figura, los valores de concentración de $\mathrm{CO}_{2}$ son bajos, al igual que los valores de $Q_{p}^{-1} / Q_{s}^{-1}$. 


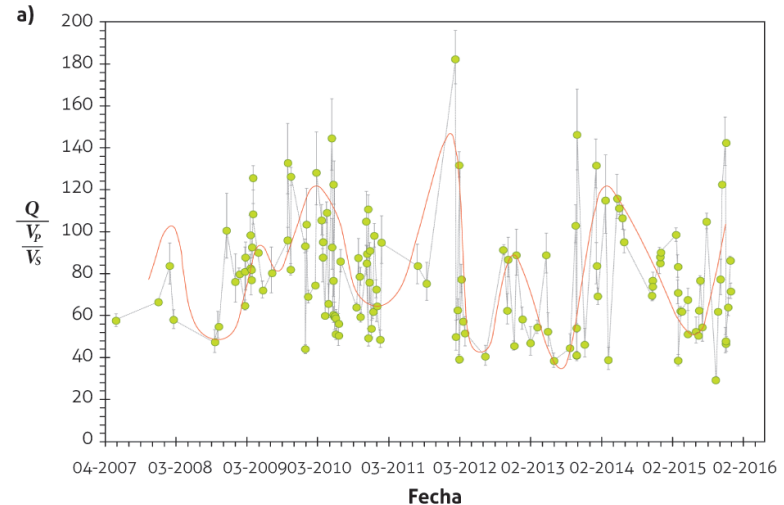

b)

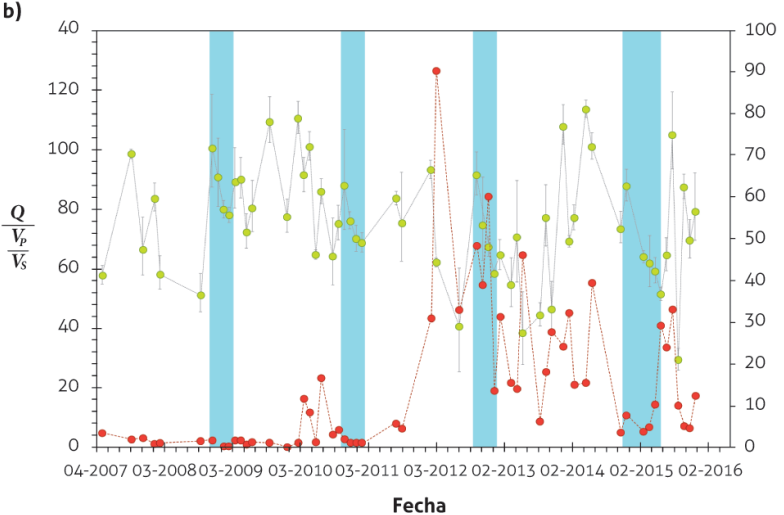

Figura 10. (a) Relación entre la variación temporal individual de la relación $Q / V / V$ (círculos amarillos) y energía sísmica radiada de sismos LP (líneas azules); la curva punteada roja representa el ajuste polinómico de los valores individuales de $Q / V / V$.

(b) la misma relación para el promedio mensual. Se muestran los valores de $Q / V / V$ en sismos de la fuente sismogénica mostrada en la figura $8 \mathrm{~b}$. Los rectángulos azules representan períodos de disminución de $Q / V_{p} / V_{s}$ en más de tres meses seguidos. Las líneas verticales sobre los picos representan el error estándar

Fuente: autores

Esto hace suponer que la actividad reciente del VP está controlada principalmente por el sistema hidrotermal, con predominio de fluidos en fase líquida. El hecho de que no se haya observado ningún cambio temporal relevante de $Q^{-1}$ durante el período estudiado concuerda con los niveles de actividad del VP, que se han mantenido bajos durante dicho período. Tales valores sirven de línea base para el futuro y para comparar con valores que se obtengan en años posteriores, y además, para tener una idea de los niveles de

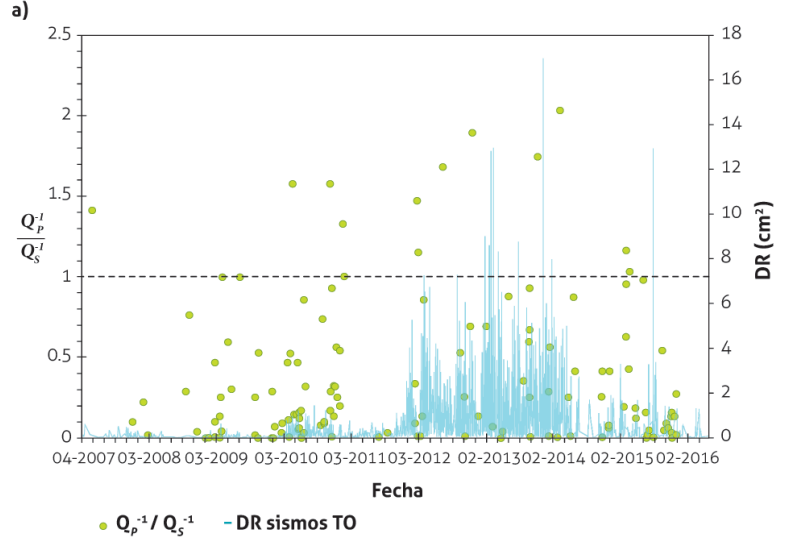

b)

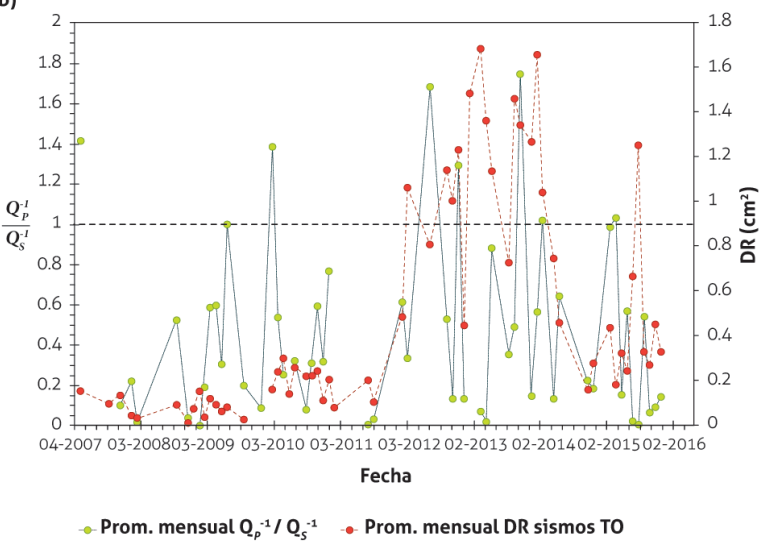

Figura 11. a) Relación entre la variación temporal individual de la relación $Q_{p}^{-1} / Q_{s}^{-1}$ de los sismos VT seleccionados y el desplazamiento reducido (DR) de sismos asociados a actividad de fluidos tipo TO en el volcán Puracé. b) Variación temporal del promedio mensual de la relación $Q_{p}^{-1} / Q^{-1}$ y el desplazamiento reducido (DR) de sismos asociados a actividad de fluidos tipo TO. La línea negra gruesa punteada representa el valor de la relación $Q_{p}^{-1} / Q_{s}^{-1}=1$

Fuente: autores

atenuación sísmica que tiene la zona del VP, que en general son intermedios $(Q=40-800$, promedio $Q=160)$, lo que sugiere la presencia de fluidos en fase líquida en su estructura, además de materiales rígidos (lavas), materiales poco consolidados, fracturados o con fluidos. Por otra parte, la relación $Q_{p}^{-1} / Q_{s}^{-1}$ permite diferenciar entre fluidos en fase líquida y fase gaseosa, lo que hace que este parámetro sea útil para el monitoreo del VP. 


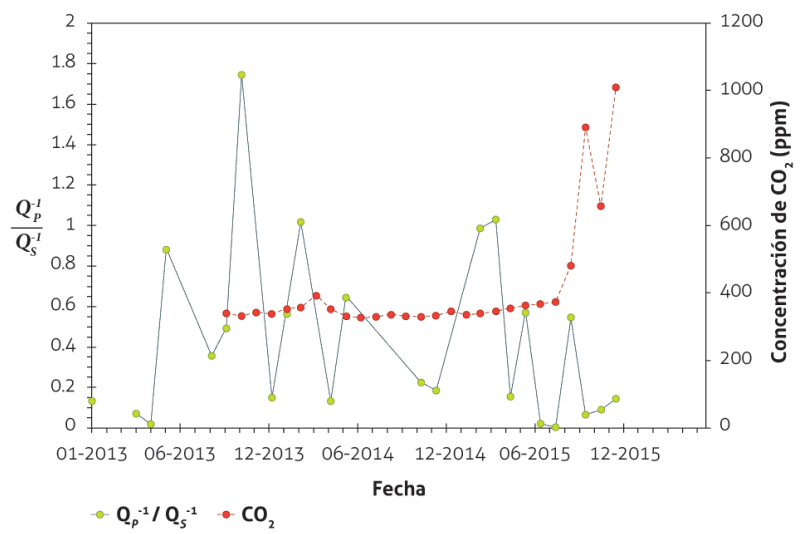

Figura 12. Serie de tiempo de la relación $Q_{p}^{-1} / Q_{s}^{-1}$ y la concentración de $\mathrm{CO}_{2}$ en la fumarola principal del cráter activo del VP para el período 2013-2016

Fuente: autores

\section{Conclusiones}

En este trabajo se analizó la variación temporal de la atenuación de ondas sísmicas en sismos volcano-tectónicos (VT) ocurridos en la zona del volcán Puracé. Las variaciones temporales de $Q_{p}^{-1} / Q_{s}^{-1}$ observadas en el VP en el período estudiado pueden estar asociadas a cambios en la actividad de su sistema hidrotermal, que probablemente ha experimentado poco aporte de gases que podrían provenir de una fuente magmática profunda, que permanece estable hasta la fecha, mientras que, en su gran mayoría, esta actividad hidrotermal está relacionada con fluidos en fase líquida, principalmente agua.

El cálculo rutinario de la relación $Q_{p}^{-1} / Q_{s}^{-1}$ a partir de valores de Coda $Q$ y de $V p / V s$ obtenidos de la observación puede servir como un indicador de la mayor o menor presencia de gas en la parte superficial del edificio volcánico. Este parámetro se puede obtener fácilmente y puede ser implementado en la rutina diaria de monitoreo volcánico. Si se tiene una línea base de suficiente duración, es posible calibrarlo para determinar diferentes niveles que pueden ser usados en conjunto con otros parámetros geoquímicos, sísmicos o de deformación para mejorar el monitoreo del volcán Puracé y de otros volcanes activos colombianos.

\section{AGRADECIMIENTOS}

Los autores desean agradecer a los compañeros del SGC y al Observatorio Vulcanológico de Popayán (OVSPo) por sus aportes y observaciones a este trabajo. Este proyecto hace parte de la actividad de la Dirección de Geoamenazas, Investigación y Monitoreo de la actividad volcánica. Dos revisores anónimos mejoraron la versión final del manuscrito con sus comentarios y sugerencias.

\section{REFERENCIAS BIBLIOGRÁFICAS}

Aki, K. y Chouet, B. (1975). Origin of coda waves: source, attenuation and scattering effects. Journal of Geophysical Research, 80, 3322-3342. Doi: 10.1029/ JB080i023p03322.

Alpala, R., Londoño, J., Torres, R. y Cadena, O. (2014). Análisis de posibles fuentes generadoras de eventos sísmicos tipo "tornillo" en el volcán Puracé, Colombia. Informe de investigación. Servicio Geológico Colombiano.

Alparone, S., Andronico, D., Lodato, L. y Sgroi, T. (2003). Relationship between tremor and volcanic activity during the Southeast crater eruption on Mount Etna in early 2000. Journal of Geophysical Research: Solid Earth, 108(B5), 2241. Doi: 10.1029/2002JB001866.

Ambeh, W. y Fairhead, J. (1989). Coda Q estimates in the Mount Cameroon volcanic region, West Africa. Bulletin of the Seismological Society of America, 79(5), 1589-1600.

Bianco, F., Castellano, M., Del Pezzo, E. e Ibáñez, J. (1999). Attenuation of short-period seismic waves at Mt. Vesuvius, Italy. Geophysical Journal International, 138(1), 67-76. Doi: 10.1046/j.1365-246x.1999.00868.x.

Chatterjee, S., Pitt, A. e Iyer, H. (1985). $\mathrm{V}_{\mathrm{p}} / \mathrm{V}_{\mathrm{s}}$ ratios in the Yellowstone National Park region, Wyoming. Journal of Volcanology and Geothermal Research, 26(3-4), 213-230. Doi: 10.1016/0377-0273(85)90057-5.

De Siena, L., Thomas, C., Waite, G., Moran, S. y Klemme, S. (2014). Attenuation and scattering tomography of the deep plumbing system of Mount St. Helens. Journal of Geophysical Research: Solid Earth, 119(11), 8223-8238. Doi: 10.1002/2014JB011372.

Del Pezzo, E., Simini, M. e Ibáñez, J. (1996). Separation of intristic and scattering $Q$ for volcanic areas: A comparison between Etna and Campi Flegrei. Journal of Volcanology and Geothermal Research, 70(3-4), 213219. Doi: 10.1016/0377-0273(95)00056-9.

Del Pezzo, E. (2008). Seismic wave scattering in volcanoes. Advances in Geophysics. New York: Academic Press. Doi: 10.1016/S0065-2687(08)00013-7. 
Dibble, R. (1974). Volcanic seismology and accompanying activity of Ruapehu Volcano, New Zealand. En Physical Volcanology. Amsterdam: Elsevier.

Espinosa, A. (2001). Erupciones históricas de los volcanes colombianos (1500-1995). Bogotá: Academia Colombiana de Ciencias Exactas, Físicas y Naturales.

Fehler, M., Roberts, P. y Fairbanks, T. (1988) A temporal change in coda wave attenuation observed during an eruption of Mount St. Helens. Journal of Geophysical Research: Solid Earth, 93(B5), 4367-4373. Doi: 10.1029/JB093iB05p04367.

Gao, L. (1992). Physical meaning of coda envelopes. En Volcanic Seismology. Berlin, Heidelberg, New York: Springer.

Giampicolo, E., D’Amico S., Patanè, D. y Gresta, S. (2007). Attenuation and source parameters of shallow microearthquakes at Mt. Etna Volcano, Italy. Bulletin of the Seismological Society of America, 97(1B), 184-197. Doi: 10.1785/0120050252.

Guo, M., Fu, L. y Ba, J. (2009). Comparison of stress-associated coda attenuation and intrinsic attenuation from ultrasonic measurements. Geophysical Journal International, 178(1), 447-456. Doi: 10.1111/j.1365246X.2009.04159.x.

Gusev, A. y Lemzikob, V. (1985). Properties of scattered elastic waves in the lithosphere of Kamchatka: Parameters and temporal variations. Tectonophysics, 112(1), 137-153. Doi: 10.1016/0040-1951(85)90177-5.

Hermana, M., Harith, Z., Sum, C. y Ghosh, D. (2014). Is the $\mathrm{Q}_{\mathrm{p}} / \mathrm{Q}_{\mathrm{s}}$ atrribute better for hydrocarbon prediction? Journal of Applied Sciences, 14(22), 3095-3100. Doi: 10.3923/jas.2014.3095.3100.

Ingeominas. (2009a). Boletín de actividad de los volcanes Nevado del Huila, Puracé y Sotará, primer semestre de 2009. Informe interno. Bogotá.

Ingeominas. (2009b). Boletín de actividad de los volcanes Nevado o del Huila, Puracé y Sotará, segundo semestre de 2009. Informe interno. Bogotá.

Ingeominas. (2010). Boletín de actividad de los volcanes Nevado del Huila, Puracé y Sotará, primer semestre de 2010. Informe interno. Bogotá.

Jin, A. y Aki, K. (1986). Temporal change in coda Q before the Tangsham earthquake of 1976 and the Haicheng earthquake of 1975. Journal of Geophysical
Research: Solid Earth, 91(B5), 665-673. Doi: 10.1029/ JB091iB01p00665.

Kumagai, H., Chouet, B. y Nakano, M. (2002). Temporal evolution of a hydrothermal system in Kusatsu-Shirane Volcano, Japan, inferred from the complex frequencies of long-period events. Journal of Geophysical Research: Solid Earth, 107(B10), ESE 9-1-ESE 9-10. Doi: 10.1029/2001JB000653.

Kvamme, L. y Havskov, J. (1989). Q in Southern Norway. Bulletin of the Seismological Society of America, 79(5), 1575-1588.

Lacruz, J., Ugalde, A., Vargas, C. y Carcolé, E. (2009). Coda-wave attenuation imaging of Galeras Volcano, Colombia. Bulletin of the Seismological Society of America, 99(6), 3510-3515. Doi: 10.1785/0120080366.

Londoño, J. (1996). Temporal change in coda Q at Nevado del Ruiz Volcano, Colombia. Journal of Volcanology and Geothermal Research, 73(1-2), 129-139. Doi: 10.1016/0377-0273(95)00084-4.

Londoño, J. y Sudo, Y. (2002). A warning model based on temporal changes of coda Q for volcanic activity at Nevado del Ruiz Volcano, Colombia. Bulletin of Volcanology, 64(5), 303-315. Doi: 10.1007/s00445-0020207-4.

Mavko, G., Dvorkin, J. y Walls, J. (2005). A theoretical estimate of $S$-wave attenuation in sediment. Conference Paper. Houston: 2005 SEG Annual Meeting.

Monsalve, M. (1996). Depósitos piroclásticos asociados a la actividad explosiva del volcán Puracé actual (Colombia). Manizales: Memorias del VIII Congreso Colombiano de Geología.

Monsalve, M. y Pulgarín, B. (1999). Cadena volcánica de los Coconucos (Colombia): centros eruptivos y producto recientes. Boletín Geológico, 37(1-3), 17-51.

Ortiz, R., Correig, A., Díez, J. y Muñoz, M. (1992). Apparent variation of coda $\mathrm{Q}$ in Phlegraean Fields during the bradyseismic crisis of 1982-1984. En Volcanic Seismology. Berlin: Springer-Verlag Berlin Heidelberg.

Phillips, W. y Aki, K. (1986) Site amplification of coda waves from local earthquakes in central California. Bulletin of the Seismological Society of America, 76(3), 627-648.

Prasad, M., Zimmer, M., Berge, P. y Bonner, B. (2004). Laboratory measurements of velocity and attenuation 
in sediments. Technical report. Society of Exploration Geophysicists. UCRL-JRNL-205155.

Pulli, J. (1984). Attenuation of coda waves in New England. Bulletin of the Seismological Society of America, 74(4), 1149-1166.

Qi, Q., Müller, T. y Pervukhina, M. (2017). Sonic $Q_{P} / Q_{S}$ ratio as diagnostic tool for shale gas saturation. Geophysics, 82(3), 97-103. Doi: 10.1190/geo2016-0499.1.

Roecker, S., Tucker, B., King, J. y Hatzfeld, D. (1982). Estimates of $\mathrm{Q}$ in Central Asia as a function of frequency and depth using the coda of locally recorded earthquakes. Bulletin of the Seismological Society of America, 72(1), 129-149.

Sato, H. (1977). Energy propagation including scattering effects sengle isotropic scattering approximation. Journal of Physics of the Earth, 25(1), 27-41. Doi: 10.4294/jpe1952.25.27.

Servicio Geológico Colombiano (SGC). (2016). Informe anual de actividad de los volcanes del segmento centro de Colombia. Informe interno. Bogotá.

Servicio Geológico Colombiano (SGC). (2017). Informe mensual de actividad de los volcanes del segmento centro de Colombia. Informe interno. Bogotá.

Singh, S. y Herrmann, R. (1983). Regionalization of crustal coda $\mathrm{Q}$ in the continental United States. Journal of Geophysical Research: Solid Earth, 88(B1), 527-538. Doi: 10.1029/JB088iB01p00527.
Tusa, G., Malone, S., Giampiccolo, E., Gresta, S. y Musumeci, C. (2004). Attenuation of short-period P-waves at Mount St. Helens. Bulletin of the Seismological Society of America, 94(4), 1441-1455. Doi: 10.1785/012003040.

Vargas, C., Pujades, L. y Caneva, A. (2012). Attenuation structure of the Galeras Volcano, Colombia. Boletin de Geología, 34(2), 149-161.

Wegler, U. y Luhr, B. (2001). Scattering behaviour at Merapi Volcano (Java) relevaled from an active seismic experiment. Geophysical Journal International, 145(3), 579-592. Doi: 10.1046/j.1365-246x.2001.01390.x.

Yamamoto, M. y Sato, H. (2010). Multiple scattering and mode conversion revealed by an active seismic experiment at Asama Volcano, Japan. Journal of Geophysical Research: Solid Earth, 115(B7). Doi: 10.1029/2009JB007109.

Zhang, Z. y Stewart, R. (2007). Seismic attenuation and well log analysis in a heavy oilfield. CREWES Research Report, 19, 1-16. Doi: 10.1190/1.3059251. 